\title{
A THEOREM ON CONVEX CONES WITH APPLICATIONS TO LINEAR INEQUALITIES ${ }^{1}$
}

JERRY W. GADDUM

1. Introduction. This note is concerned with the convex cone associated with the two systems of linear inequalities

$$
\sum_{j=1}^{n} a_{i j} x_{j} \geqq 0, \quad i=1,2, \cdots, m,
$$

and

$$
\sum_{j=1}^{n} a_{i j} x_{j} \geq 0, \quad i=1,2, \cdots, m,
$$

where the symbol $\geqq$ demands that the inequality $(>)$ hold for at least one value of $i$. For brevity these systems will be written (1) $A x \geqq 0$ and (2) $A x \geq 0$.

Interpreting $\left(a_{i 1}, \cdots, a_{i n}\right)$ as a vector $a_{i}$ in $E_{n}$, with initial point at the origin, we denote by $A$ the convex cone generated by these $m$ vectors and by $A^{*}$ the polar cone, the vectors of which give the solutions of (1).

The purpose of this paper is to show that in general $A \cdot A^{*} \neq 0$ and to characterize the cases in which $A$ and $A^{*}$ do intersect in the null vector. Some applications are then made to obtain theorems on the existence of, and methods of obtaining, solutions of (1) and (2).

2. The main theorem. Before proving the main theorem, two remarks are appropriate. First, the writer has previously given an entirely different proof of a special case of the theorem, in which it was assumed that $A$ is not contained in an $E_{n-1}$. The statement of this case may be found in [2]. ${ }^{2}$ It is clear that Theorem 2.1 follows immediately from this special case. In [6] a stronger version has been proved under the assumption that $A$ does not contain a straight line. Second, it will be seen that the proof given here does not require that the cone $A$ be generated by finitely many vectors, but the applications to be made require this.

At the suggestion of the referee, we state and prove the theorem in a more general setting than is needed for the applications. The proof

Received by the editors March 25, 1952.

${ }^{1}$ The preparation of this paper was sponsored (in part) by the Office of Scientific Research, USAF.

2 Numbers in brackets refer to the bibliography. 
given is substantially that of the referee. If $E$ is an inner-product space and $A$ a convex cone with vertex 0 in $E, A^{*}$ denotes the set of $x \in E$ such that $(x, a) \geqq 0$ for all $a \in A$.

THEOREM 2.1. If $E$ is a complete inner-product space and $A$ a convex cone of $E$ with vertex 0 , then $A \cdot A^{*}=0$ if and only if $A$ is a linear subspace of $E$.

Proof. If $A$ is a linear subspace, $A^{*}$ is its orthogonal complement. If $A$ is not a linear subspace, there is an $a \in A$ for which $-a$ is not in $A$. If $b$ is the nearest point of $A$ to $-a$, let $c=a+b$. Then $c \in A$.

Now the hyperplane through $b$, orthogonal to $c$, is a supporting plane of $A$, for otherwise $A$ would have an element closer than $b$ to $-a$. In other words, $(y, c) \geqq(b, c)$, for each $y$ of $A$. In particular $(2 b, c)=2(b, c) \geqq(b, c)$ and $(0, c)=0 \geqq(b, c)$. Thus $(b, c)=0$ and $b \in A^{*}$, proving the theorem.

We might pause at this point to mention a method of obtaining solutions of (2). If the rows of the matrix $A$ form a set $A_{0}$, we may adjoin the sum of each two, obtaining a set $A_{1}$, repeat the process, obtaining $A_{2}$, and so on, obtaining vectors arbitrarily close to a solution.

3. Some existence theorems. In this section the foregoing discussion is applied to obtain theorems on the existence of solutions for a system of linear inequalities. A vector $y$ is called positive if $y_{i}>0$ for each $i$, non-negative if $y_{i} \geqq 0$ for each $i$ and $y_{i}>0$ for some $i$.

Theorem 3.1. The system (2) has a solution if and only if $A \cdot A^{*} \neq 0$.

The proof being immediate from Theorem 2.2, we state the same result algebraically.

Corollary. If (2) has a solution, it has one of the form $x=A^{\prime} y$, where $y$ is non-negative and $A^{\prime}$ is the transpose of the matrix $A$.

TheOREM 3.2. In order that (2) have a solution, it is necessary and sufficient that the system $A A^{\prime} y \geq 0$ have a non-negative solution.

Proof. First, if $A A^{\prime} y \geq 0$ has any solution at all, the relation $x=A^{\prime} y$ gives a solution of $A x \geq 0$. On the other hand, if $A x \geq 0$ has a solution, the corollary to Theorem 3.1 states that it has one of the form $x=A^{\prime} y, y$ non-negative, and this $y$ is a solution of $A A^{\prime} y \geq 0$.

It should be observed that this theorem is a strengthening of the result, in [1], that $A x \geq 0$ and $A A^{\prime} y \geq 0$ are either both consistent or both inconsistent. 
CoROllaRY. If $B$ is a symmetric positive semi-definite matrix, By $\geq 0$ has a solution if and only if it has a non-negative one.

Corollary. A sufficient condition that (1) have a nontrivial solution is that $A A^{\prime} y \geq 0$ have a (non-negative) solution.

CoROLlaRY. If the rank of $A$ is $n$, a necessary condition that (1) have a nontrivial solution is that $A A^{\prime} y \geq 0$ have a (non-negative) solution.

In [3] and [7] a necessary and sufficient condition is given that a system of type (2) be solvable. Using Theorem 2 of [3] we can state the following theorem.

TheOREM 3.3. $A A^{\prime} y \geq 0$ (and hence (2)) has a solution if and only if $A A^{\prime} y=0$ has no positive solution.

In [4] a method is given for determining whether or not a system of homogeneous linear equations has a positive solution. In the form given, however, the method does not seem feasible for purposes of computation.

An obvious necessary condition that $A A^{\prime} y=0$ have a positive solution is that for any collection of rows of $A A^{\prime}$, the $m$ column sums be all zero or have a pair of terms of opposite sign. Hence a sufficient condition that $A A^{\prime} y \geq 0$ (and hence (2)) have a solution is that for some collection of rows of $A A^{\prime}$, the $n$ column sums form a non-negative set.

Having obtained a sufficient condition that $A A^{\prime} y \geq 0$ be solvable, we now derive a necessary condition. Denoting $A A^{\prime}$ by $B=\left(b_{i j}\right)$, we know by Theorem 3.2 that if $B y \geq 0$ has a solution it has a nonnegative one. Hence if $B y \geq 0$ has a solution, then the system

$$
\begin{aligned}
& b_{11} y_{1}+\cdots+b_{1 m} y_{m} \geq 0 \text {, } \\
& b_{m 1} y_{1}+\cdots+b_{m m} y_{m} \geq 0 \text {, } \\
& y_{1} \quad \geq 0 \text {, } \\
& y_{m} \geq 0
\end{aligned}
$$

also has a solution. Using the theorem just quoted from [3] and [7], this has a solution if and only if the system of equations

$$
\begin{aligned}
& b_{11} u_{1}+\cdots+b_{m 1} u_{m}+u_{m+1}=0 \text {, } \\
& b_{1 m} u_{1}+\cdots+b_{m m} u_{m}+u_{2 m}=0
\end{aligned}
$$


has no positive solution. In other words we have proved

Theorem 3.4. A necessary condition that $A A^{\prime} y \geq 0$ (and hence (2)) have a solution is that $-A A^{\prime} y=0$ have no positive solution.

In Theorem D 5, p. 50, of [5], a criterion is given for the existence of positive solutions of $-B y=0$.

\section{BIBLIOGRAPHY}

1. S. Agmon, The relaxation method for linear inequalities, Prepublication Copy, National Bureau of Standards, Los Angeles, California.

2. L. M. Blumenthal, Metric methods in linear inequalities, Duke Math. J. vol. 15 (1948) pp. 955-966.

3. L. L. Dines, Note on certain associated systems of linear equalities and inequalities, Ann. of Math. (2) vol. 28 (1926-27) pp. 41-42.

4. - On positive solutions of a system of linear equations, Ann. of Math. (2) vol. 28 (1926-27) pp. 386-392.

5. T. S. Motzkin, Beiträge zur Theorie der linearen Ungleichungen, Dissertation, Basel, 1933, Jerusalem, 1936.

6. - Linear inequalities, Mimeographed Lecture Notes, University of California, Los Angeles, 1951.

7. E. Stiemke, Über positive Lösungen homogener linearer Gleichungen, Math. Ann. vol. 76 (1915) pp. 340-342.

National Bureau of Standards, Los Angeles 